\title{
Homotopy perturbation method and seventh-order boundary value problems
}

\author{
Fazle Mabood $^{1, *}$, Muhammad Idrees ${ }^{2}$ \\ ${ }^{I}$ Department of Mathematics, Edwardes College Peshawar, KPK 25000, Pakistan \\ ${ }^{2}$ Department of Mathematics, City University Peshawar, KPK 25000, Pakistan \\ *Corresponding author E-mail: mabood1971@yahoo.com
}

\begin{abstract}
Homotopy Perturbation Method (HPM) is employed to find the approximate solution of seventh-order linear and nonlinear boundary value problems. The effectiveness of HPM is illustrated through two examples. The approximate solution of the equations is obtained in terms of convergent series. Interestingly a high accurate result was obtained with only one $p$-term of the series using HPM. This thus confirms the strength of HPM. Low absolute error indicates that HPM is a promising tool for solving higher order linear and nonlinear boundary value problems.
\end{abstract}

Keywords: Error Estimate, Homotopy Perturbation Method, Seventh-Order BVP.

\section{Introduction}

Consider the seventh-order boundary value problem:

$$
u^{(7)}=f(x, u(x)), \quad a \leq x \leq b
$$

with boundary conditions

$$
\begin{array}{ll}
u^{(i)}=A_{i}, & i=0,1,2,3 \\
u^{(j)}=B_{j}, & j=0,1,2
\end{array}
$$

Various numerical and semi-analytical methods have been introduced for the solution of high order boundary value problems. The aim of the present work is to extend homotopy perturbation method to solve seventh-order boundary value problems which is in fact the coupling of traditional perturbation method and homotopy method proposed by He [1-11]. HPM deforms difficult problem into a set of problems which are easy to handle by Ganji and coworkers [12-14]. In this method the solution is considered as the summation of an infinite series that usually converges rapidly to the exact solutions by Abbasbanday [15]. The analytical solution for seventh-order differential equations (which are rarely exists in open literature) obtained via HPM is shown to be in good agreement with exact solution. This study evidently indicates that the proposed method may be considered as an alternative technique for solving linear and nonlinear problems in science and engineering.

\section{Basic Idea of HPM}

We review the basic idea of HPM [1-15].

Consider the following differential equation and boundary condition:

$$
A(u)-f(r)=0, \quad r \in \Omega
$$

with boundary conditions: 


$$
B\left(u, \frac{\partial u}{\partial n}\right)=0, \quad r \in \Gamma
$$

Where $A, B, f(r), \Gamma$ are a general differential, a boundary operator, a known analytical function and the boundary of the domain $\Omega$, respectively. Generally speaking, the operator $A$ can be divided into a linear part $L$ and a nonlinear part $N(u)$. So Eq. (3) can be written as:

$$
L(u)+N(u)-f(r)=0
$$

By the homotopy method, we construct a homotopy $v(r, p): \Omega \times[0,1] \rightarrow R$ which satisfies:

$$
H(v, p)=(1-p)\left[L(v)-L\left(u_{0}\right)\right]+p[A(v)-f(r)]=0, \quad p \in[0,1], r \in \Omega
$$

or

$$
H(v, p)=L(v)-L\left(u_{0}\right)+p L\left(u_{0}\right)+p[N(v)-f(r)]=0
$$

Where $p \in[0,1]$ is an embedding parameter, while $u_{0}$ is an initial approximation of Eq. (3) which satisfy the boundary conditions. Obviously, from Eqs. (6), (7) we obtain:

$$
\begin{aligned}
& H(v, 0)=L(v)-L\left(u_{0}\right)=0 \\
& H(v, 1)=A(v)-f(r)=0
\end{aligned}
$$

The changing process of $p$ from zero to unity is just that of $v(r, p)$ from $u_{0}$ to $u(r)$. In topology, it is called deformation, while $L(v)-L\left(u_{0}\right)$ and $A(v)-f(r)$ are called homotopy. According to the HPM, we can use embedding parameter $p$ as a "small parameter", and assume that the solutions of Eqs. (6) and (7) can be written as a power series in $p$ :

$$
v=v_{0}+p v_{1}+p^{2} v_{2}+\ldots
$$

Setting $p=1$ yields in the approximate solution of Eq. (10) to:

$$
u=\lim _{p \rightarrow 1} v=v_{0}+v_{1}+v_{2}+\ldots
$$

The combination of the perturbation method and the homotopy method is known as HPM, which eliminates the drawbacks of the traditional perturbation methods while keeping all its advantages. The series (11) is convergent for most cases. However, the convergent rate depends on the nonlinear operator $N(v)$. Further, He [1] made the following suggestions:

(1) The second derivative of $N(v)$ with respect to $v$ must be small because the parameter may be relatively large, i.e. $p \rightarrow 1$.

(2) The norm of $L^{-1} \frac{\partial N}{\partial v}$ must be smaller than one so that the series converges.

\section{Numerical Examples}

In order to illustrate the ability of HPM in solving seventh-order problems, we consider the following two examples herein:

\subsection{Example 1}

Consider the seventh-order linear boundary value problem. 


$$
\frac{d^{7} u}{d x^{7}}=u-7 e^{x}
$$

with boundary conditions:

$$
\begin{aligned}
& u(0)=1, u^{(1)}(0)=0, u^{(2)}(0)=-1, u^{(3)}(0)=-2, \\
& u(1)=0, u^{(1)}(1)=-e, u^{(2)}(1)=-2 e
\end{aligned}
$$

The analytic solution of Eq. (12) is $u(x)=e^{x}(1-x)$.

Applying the proposed method (HPM) on Eq. (12), a homotopy can be constructed as follows:

$$
H(v, p)=(1-p)\left(\frac{d^{7} u}{d x^{7}}-\frac{d^{7} u_{0}}{d x^{7}}\right)+p\left(\frac{d^{7} u}{d x^{7}}-u+7 e^{x}\right)=0
$$

Substituting $u=u_{0}+p u_{1}+\ldots$ in Eq. (14) and rearranging the resultant equation based on powers of $p-$ terms, one has:

$$
\begin{array}{ll}
p^{0}: & \frac{d^{7} u_{0}}{d x^{7}}=0 \\
p^{1}: & \frac{d^{7} u_{1}}{d x^{7}}+7 e^{x}-u_{0}=0 \\
p^{2}: & \frac{d^{7} u_{2}}{d x^{7}}-u_{1}
\end{array}
$$

with boundary conditions:

$$
\begin{aligned}
& \begin{cases}u_{0}(0)=1, & u_{0}(1)=0, \\
u_{0}{ }^{(1)}(0)=0, & u_{0}^{(1)}(1)=-e \\
u_{0}^{(2)}(0)=-1, & u_{0}^{(2)}(1)=-2 e \\
u_{0}^{(3)}(0)=-2\end{cases} \\
& \left\{\begin{array}{ll}
u_{j}(0)=0, & u_{j}(1)=0, \\
u_{j}^{(1)}(0)=0, & u_{j}^{(1)}(1)=0 \\
u_{j}^{(2)}(0)=0, & u_{j}^{(2)}(1)=0 \\
u_{j}^{(3)}(0)=0 &
\end{array} \quad j=1,2, \ldots\right.
\end{aligned}
$$

With the effective initial approximation for $u_{0}$ from the conditions Eq. (18), the solution of Eq. (15) may be written as follows:

$$
u_{0}(x)=\frac{1}{6}\left(6-3 x^{2}-2 x^{3}-66 x^{4}+24 e x^{4}+114 x^{5}-42 e x^{5}-49 x^{6}+18 e x^{6}\right)
$$

By similar approach, the rest of components were obtained.

According to the HPM, we can conclude that:

$$
u(x)=\lim _{p \rightarrow 1} u(x)=u_{0}(x)+u_{1}(x)+\ldots
$$

Therefore, substituting the value of $u_{0}(x)$ from Eq. (19) into Eq. (20) yields:

$$
u(x)=\frac{1}{6}\left(6-3 x^{2}-2 x^{3}-66 x^{4}+24 e x^{4}+114 x^{5}-42 e x^{5}-49 x^{6}+18 e x^{6}\right)
$$


Comparison of approximate analytical solution using HPM with exact solution is tabulated in Table 1 along with absolute error of the HPM, showing a remarkable agreement. It is noteworthy that even higher accuracy could be achieved by solving second and third terms from Eqs. (16) and (17).

Table 1: Comparison of solutions using HPM with Exact solution

\begin{tabular}{cccc}
\multicolumn{4}{c}{ Table 1: Comparison of solutions using HPM with Exact solution } \\
\hline$x$ & HPM & Exact & Absolute Error \\
\hline 0.0 & 1 & 1 & 0 \\
0.1 & 0.99465 & 0.99465 & $1.32 \times 10^{-13}$ \\
0.2 & 0.97712 & 0.97712 & $1.55 \times 10^{-12}$ \\
0.3 & 0.94490 & 0.94490 & $5.52 \times 10^{-12}$ \\
0.4 & 0.89509 & 0.89509 & $1.14 \times 10^{-11}$ \\
0.5 & 0.82436 & 0.82436 & $1.67 \times 10^{-11}$ \\
0.6 & 0.72884 & 0.72884 & $1.83 \times 10^{-11}$ \\
0.7 & 0.60412 & 0.60412 & $1.46 \times 10^{-11}$ \\
0.8 & 0.44510 & 0.44510 & $7.56 \times 10^{-13}$ \\
0.9 & 0.24595 & 0.24595 & $1.52 \times 10^{-13}$ \\
1.0 & 0 & 0 & 0 \\
\hline
\end{tabular}

\subsection{Example 2}

Consider the seventh-order nonlinear boundary value problem.

$$
\frac{d^{7} u}{d x^{7}}=u^{2} e^{-x}
$$

with boundary conditions:

$$
\begin{aligned}
& u(0)=u^{(1)}(0)=u^{(2)}(0)=u^{(3)}(0)=1, \\
& u(1)=u^{(1)}(1)=u^{(2)}(1)=e
\end{aligned}
$$

The analytic solution of Eq. (22) is $u(x)=e^{x}$.

Applying the proposed method (HPM) on Eq. (22), a homotopy can be constructed as follows:

$$
H(v, p)=(1-p)\left(\frac{d^{7} u}{d x^{7}}-\frac{d^{7} u_{0}}{d x^{7}}\right)+p\left(\frac{d^{7} u}{d x^{7}}-u^{2} e^{-x}\right)=0
$$

Substituting $u=u_{0}+p u_{1}+\ldots$ in Eq. (24) and rearranging the resultant equation based on powers of $p-$ terms, one has:

$$
\begin{array}{ll}
p^{0}: & \frac{d^{7} u_{0}}{d x^{7}}=0 \\
p^{1}: & \frac{d^{7} u_{1}}{d x^{7}}-e^{-x} u_{0}^{2}=0 \\
p^{2}: & \frac{d^{7} u_{2}}{d x^{7}}-2 e^{-x} u_{0} u_{1}=0
\end{array}
$$

with boundary conditions:

$$
\begin{cases}u_{0}(0)=1, & u_{0}(1)=e, \\ u_{0}^{(1)}(0)=1, & u_{0}^{(1)}(1)=e, \\ u_{0}^{(2)}(0)=1, & u_{0}^{(2)}(1)=e, \\ u_{0}^{(3)}(0)=1 & \end{cases}
$$




$$
\left\{\begin{array}{ll}
u_{j}(0)=0, & u_{j}(1)=0, \\
u_{j}^{(1)}(0)=0, & u_{j}^{(1)}(1)=0 \\
u_{j}^{(2)}(0)=0, & u_{j}^{(2)}(1)=0 \\
u_{j}^{(3)}(0)=0 &
\end{array} \quad j=1,2, \ldots\right.
$$

With the effective initial approximation for $u_{0}$ from the conditions Eq. (28), the solution of Eq. (25) may be written as follows:

$$
u_{0}(x)=\frac{1}{6}\left(6+6 x+3 x^{2}+x^{3}-171 x^{4}+63 e x^{4}+261 x^{5}-96 e x^{5}-106 x^{6}+39 e x^{6}\right)
$$

By similar approach, the rest of components can be obtained.

According to the HPM, we can conclude that:

$$
u(x)=\lim _{p \rightarrow 1} u(x)=u_{0}(x)+u_{1}(x)+\ldots
$$

Therefore, substituting the value of $u_{0}(x)$ from Eq. (29) into Eq. (30) yields:

$$
u(x)=\frac{1}{6}\left(6+6 x+3 x^{2}+x^{3}-171 x^{4}+63 e x^{4}+261 x^{5}-96 e x^{5}-106 x^{6}+39 e x^{6}\right)
$$

Comparison of approximate analytical solution using HPM with exact solution is tabulated in Table 2 along with absolute error of the HPM, showing a remarkable agreement. Once again, as stated in example 1, it is obvious that high accuracy could be achieved by solving Eqs. (26) and (27).

Table 2: Comparison of solutions using HPM with Exact solution

\begin{tabular}{cccc}
\hline$x$ & HPM & Exact & Absolute Error \\
\hline 0.0 & 1 & 1 & 0 \\
0.1 & 1.1051 & 1.1051 & $2.15 \times 10^{-8}$ \\
0.2 & 1.2214 & 1.2214 & $2.45 \times 10^{-7}$ \\
0.3 & 1.3498 & 1.3498 & $8.42 \times 10^{-7}$ \\
0.4 & 1.4918 & 1.4918 & $1.69 \times 10^{-6}$ \\
0.5 & 1.6487 & 1.6487 & $2.42 \times 10^{-6}$ \\
0.6 & 1.8221 & 1.8221 & $2.61 \times 10^{-6}$ \\
0.7 & 2.0137 & 2.0137 & $2.06 \times 10^{-6}$ \\
0.8 & 2.2255 & 2.2255 & $1.05 \times 10^{-6}$ \\
0.9 & 2.4596 & 2.4596 & $2.14 \times 10^{-7}$ \\
1.0 & 2.7182 & 2.7182 & $4.44 \times 10^{-16}$ \\
\hline
\end{tabular}

\section{Conclusion}

In this paper, homotopy perturbation method has been utilized for the solution of seventh-order linear and nonlinear boundary value problems. The obtained approximate analytical solution using homotopy perturbation method is then compared with exact solution. Highly accurate result has been obtained in both examples by considering only single $p$-term. It can be concluded that homotopy perturbation method is a promising tool for solving high-order boundary value problems in various fields of science and engineering.

\section{References}

[1] J.H. He, Homotopy Perturbation Technique, Applied Mech. Eng., 178(3-4) (2003) 257-262.

[2] J.H. He, Homotopy perturbation method: a new nonlinear analytical technique, Applied Mathematics and Computation, 135 (2003) $73-79$.

[3] J.H. He, Asymptotology by homotopy perturbation method, Applied Mathematics and Computation, 156(3) (2004) 591-596.

[4] J.H. He, The homotopy perturbation method for nonlinear oscillators with discontinuities, Applied Mathematics and Computation, 151 (2004) 287-292.

[5] J.H. He, Application of homotopy perturbation method to nonlinear wave equations, Chaos, Solitons \& Fractals 26 (2005) 695-700. 
[6] J.H. He, Homotopy perturbation method for bifurcation of nonlinear problems, Int. J. Nonlinear Sci. Numer. Simul., 6 (2005) $207-208$.

[7] J.H. He, Some asymptotic methods for strongly nonlinear equations, Int. J. Modern Physics B, 20 (2006) 1141-1199.

[8] J.H. He, Non-perturbative methods for strongly nonlinear problems, Dissertation, de-Verlag im Internet GmbH, Berlin. (2006)

[9] J.H. He, Comparison of homotopy perturbation method and homotopy analysis method, Applied Mathematics and Computation, 156(2) (2004) 527-539.

[10] J.H. He, An elementary introduction to the homotopy perturbation method, Computers \& Mathematics with Applications, 57(3) (2009) 410 412 .

[11] J.H. He, Recent development of the homotopy perturbation method, Topological Methods in Nonlinear Analysis, 31(2) (2008) 205-209.

[12] D.D. Ganji, M. Nourollahi, E. Mohseni, Application of He's methods to nonlinear chemistry problems, Computers and Mathematics with Applications, 54 (2007) 1122-1132.

[13] A. Rajabi, D.D. Ganji, H. Taherian, Application of homotopy perturbation method in nonlinear nonlinear heat conduction and convection equations, Physics Letters A, 360 (2007) 570-573.

[14] M. Rafei, H. Daniali, D.D. Ganji, H. Pashaei, Solution of the prey and predator problem by homotopy perturbation method, Applied Mathematics and Computation, 188 (2007) 1419-1425.

[15] S. Abbasbanday, Numerical solutions of the integral equations: Homotopy perturbation method and Adomian's decomposition method, Applied Mathematics and Computation, 173 (2006) 493-500. 